\title{
ABAODV: An Accumulator Based Ad Hoc on Demand Distance Vector Routing Protocol for Vehicular Ad Hoc Networks
}

\author{
Anush Baskaran, Sushant Ramesh, Ronnie D. Caytiles* and N.Ch.S.N Iyengar \\ VIT University, Vellore-632014, Tamilnadu, India \\ *Hannam University, Korea \\ \{anush.baskaran2013,sushant.ramesh2013,nchsniyr\}@vit.ac.in, rdcaytiles@gmail.com
}

\begin{abstract}
It is well-known, that one of the key characteristics of a Vehicular Ad-hoc Network (VANET) is the randomized movement of nodes (in this case, automobiles). For this to transpire, the routing protocols in the VANET have to swiftly respond to the network topology change with the purpose of guaranteeing successful data packet delivery. Hence, multiple routing pathways are therefore arranged in order to improve on reliability and on limited bandwidth which further lead to advanced transmission. In this paper, a minor modification has been made to the AODV routing protocol in the form of an accumulator, which inherently, helps to reduce the number of data packets present in the network at any instant which consequently, decreases the data traffic and eases the congestion in any network. VANETs have been receiving greater attention from both, industries as well as academic researchers and our proposed idea will help to improve its efficiency.
\end{abstract}

Keywords: VANET, AODV, Packet Delivery, Reliability, Accumulator, Data Traffic

\section{Introduction}

An ad-hoc network is a local area network (LAN) which is established when devices with networking capability connect without any infrastructure. These kinds of networks are self-organizing and adaptive. Rather than being dependent on a centralized system to synchronize the transmission of messages or packets of data from one node to another, the distinct network nodes transfer packets among one another. Hence, the broadcasting of data in an ad-hoc network is extemporaneous.

Several studies have been published on the analysis of VANETs as well as on the various versions of the AODV routing protocol, each with its own advantageous characteristics. Vehicular ad-hoc network is an exceedingly utilized technology with a lot of real-world applications and is a sub-category of Mobile ad-hoc network. VANETs, identical to MANETs signify the purpose of offering an important means of communication among an arbitrarily formed group of automobiles as well as between vehicles and road side equipment. The rapidly varying network topology and severely changing communication settings deploy one of the key concepts of MANETS that is a highly continuous fluctuation in vehicular motion, thus providing a challenging environment for the routing protocol employed on VANETs.

AODV is the Ad-hoc On-demand Distance Vector routing protocol which is a reactive routing protocol (as it works "on-demand"). In AODV, when data packets are sent from a source node to a destination node, it first starts a route detection process known as route discovery (RD). Through this process, the source node initially broadcasts a Route Request (RREQ) packet to all its neighboring nodes. Those neighbor nodes have no knowledge of an active route to the destination node, further broadcast the RREQ packet to their neighbors until an active route is identified or the until the maximum hop count is reached. When an intermediate node knows of an active route to said destination node, it propels a Route Reply (RREP) packet back to source node in unicast mode, via the same 
route. Ultimately, the source node collects the RREP packet and opens the route. This way, AODV decreases the number of broadcasts by creating routes based on demand. AODV also works better on larger networks than most other reactive protocols as it has a lower overhead and a higher bandwidth.

With the addition of an accumulator, the data traffic in the network can considerably be reduced. The accumulator is a sort of data structure which stores the data packets along with their source and destination nodes, without repetition. This way, there is no replication of data packets in the network and the amount of free data packets in the network is decreased, to the benefit of the users as there is lesser of the network breaking down.

Hence, the proposed idea employs an existing routing protocol, AODV, in a Vehicular Ad-hoc network with a small modification to increase the efficiency and productivity wherein reliability and throughput are directly improved.

\section{Literature Survey}

Recently, various on demand protocols have been proposed due to their low routing overheads which in turn reduce the frequencies of route re-establishment and route queries. In such protocols we notice that routes are established between the source and destination only when the source demands so.

Abolfazl Akbari [1] et.al proposed a new route maintenance algorithm based on AODV to avoid route breaks and to reestablish new routes before route breaks can occur.

C.perkins [3] et.al. explained the various subtleties in the working of the AODV algorithm as well as its capability to maintain most of the advantages of basic distance vector routing mechanisms. They also explain how the algorithm is suitable for a dynamic, self-starting network.

Himanshu Saini [5] et.al simulated the AODV algorithm on a real world mobility models for VANET using SUMO, MOVE and NS2. They also analyzed the performance of AODV with respect to various factors based on the simulation results.

J. Broch [6] et.al presented the results of a meticulous packet-level simulation comparing four multi-hop wireless ad hoc network routing protocols, namely, DSDV, TORA, DSR, and AODV.

Jerome Haerri [7] et.al present and describe VanetMobiSim, a freely available generator of accurate vehicular movement for telecommunication network simulators. They are able to validate it by illustrating how the collaboration between featured macroand micro-mobility is able to produce real world phenomena of vehicular traffic.

Jerome Haerri [8] et.al propose a framework for generating certain vehicular mobility models that comprise all realistic parameters vehicles experience while on the road, and finally propose two mobility models which are derived.

Kapil Bhagchandani [9] et.al present an efficient cluster based routing approach in order to increase overall network throughput and decrease end to end delay by maintaining an effective route for data transmission.

Kevin C. Lee [10] et.al present a survey of the various routing protocols in VANET by dividing them into their two main classifications i.e. topology based routing protocols and position based routing protocols. It also exhibits certain issues that are faced and can be overcome by further research.

M. Abolhasan [11] et.al deliver an overview with a performance comparison of an extremely wide range of routing protocols such as DSDV, WRP, GSR, FSR, STAR, DREAM, MMWN, CGSR, HSR, OLSR and TBRPF.

Marwa Altayeb [12] et.al provides general outlines and goals of VANETs with a summary of the VANET routing protocols (based on two classification forms). They also review the strengths and drawbacks of each form as well as the current trends and challenges faced in VANETs. 
Rasha Kaiss Aswed [16] et.al evaluated the performance of three diverse routing protocols in VANETs i.e. AODV, OLSR and DYMO using two different MAC protocols which are IEEE 802.11p and IEEE 802.11g. They showed that AODV outperformed the other two protocols in terms of throughput.

Sven Jaap [19] et.al developed a realistic city mobility model to evaluate the performance of four routing protocols in urban traffic scenarios. They concluded that while TORA is completely unsuitable for vehicles in the city environment, FSR, DSR and AODV showed favorable outcomes in the same scenario.

T. Camp [20] et.al describe various mobility models several of which are entity based and several of which are group mobility models. The purpose of this paper was to offer researcher a wide range of mobility models in order to make an informed decision while choosing a mobility model for a performance evaluation.

T.H. Clausen [22] et.al focus mainly on the description of OLSR, a proactive protocol. They evaluate its performance through exhaustive simulations in NS2, while comparing it with other routing protocols, particularly, two reactive protocols, DSR and AODV, under varying conditions.

\section{Routing Protocols}

The main purpose of a routing protocol is to adopt a certain method of data exchange between any two communication units. It involves the procedure in forming a route, decisions regarding the forwarding of packets, and maintenance or recovery in case there is any sort of route failure. One of the main reasons we use routing protocols is to find an ideal route between any two nodes in a network generating maximum throughput and minimum overhead.

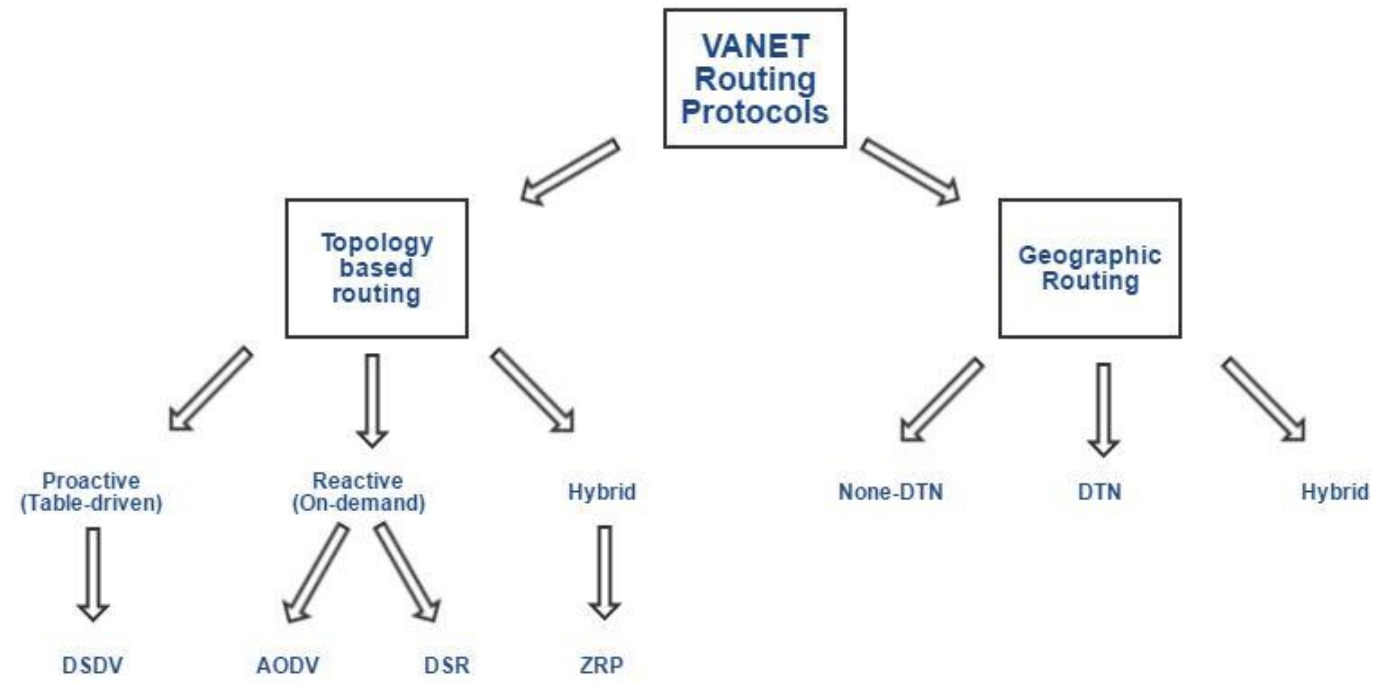

Figure 1. Classification of Routing Protocols

It is greatly challenging to design an appropriate routing protocol for VANETS. Due to the brisk, random motion of the nodes, it is not easy to come up with a routing protocol that can discover competently routing pathways among vehicles. There have been a number of routing protocols that have been developed with algorithms, primarily for MANET. Many of these algorithms have also been modified and enhanced, but they still cannot attain the same degree of adeptness for VANET. These routing protocols are 
characterized according to the various aspects they possess. Shown in Figure 1 is the classification of routing protocols along with a few examples.

\subsection{Ad-hoc On-demand Distance Vector (AODV) Routing Protocol}

As is now clear, a proactive based protocol cannot be suited to the rapid node mobility of the VANET. Proactive based routing protocols are table-driven and the volume of table information may become extremely large due to the node movement and this could lead to a heavy utilization of bandwidth. AODV, on the other hand is a reactive based routing protocol which creates routes in the network only when a packet is to be sent from one node to another. Here, nodes can detect other nodes in the network by various methods, the most common of which is by sending broadcast messages to the nodes in its immediate neighborhood (i.e. the neighboring nodes/vehicles). The two main goals of the AODV routing protocol are:

- To create routes only when required.

- To detect and spread information about variations in the network, mainly that of node positions.

There are five basic steps involved in the working of the AODV routing protocol, which are: path discovery, reverse path setup, forward path setup, route table management and path maintenance.

3.1.1. Path Discovery: Each node in a network has two key data fields: a node sequence number and a broadcast ID. When a node (i.e. source node) wants to communicate with another node in the network (i.e. destination node) about which it holds no routing information, the path discovery process begins.
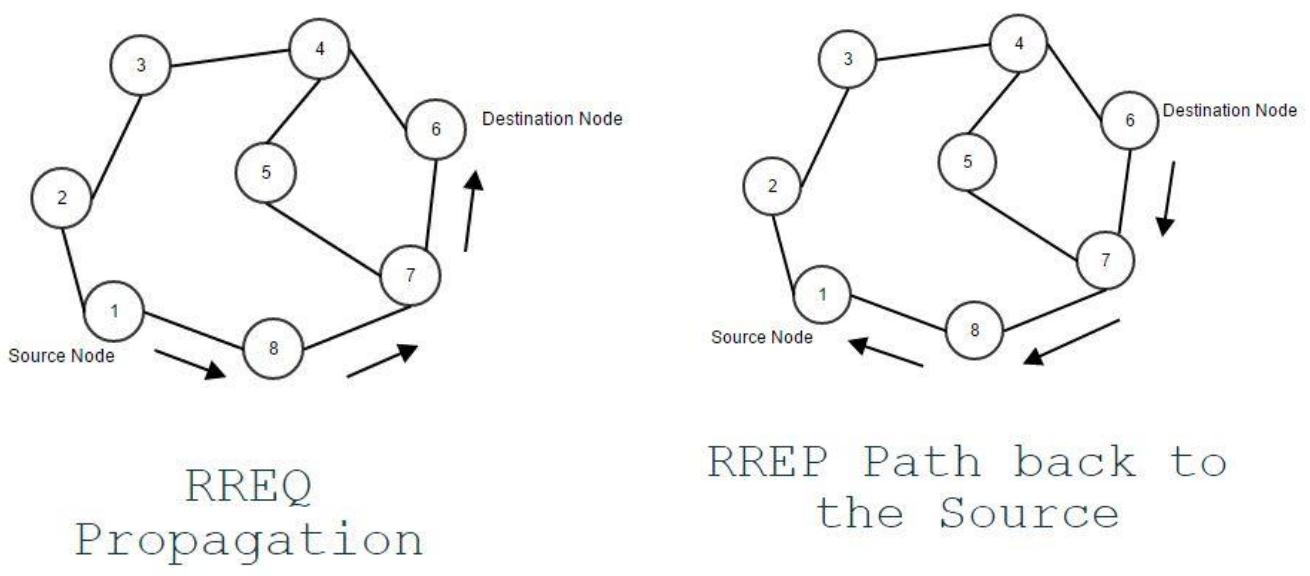

Figure 2.1(a). RREQ Packets for Route Discovery;2.1(b). RREP Packets after
path discovered

Firstly, a RREQ (route request) packet is broadcasted from the source node to all its immediate neighbors.

This RREQ packet contains information about both, the source and destination node. Each node that receives the RREQ can do one of two things. If the destination node has been reached, (as shown in Figure 2.1) it can fulfill the RREQ by sending an RREP (route reply) packet back to the source. 
This is done by unicast method (shown in Figure 2.2). Also, it drops the RREQ packet in order to make the communication completely loop-free. If the node receiving the packet is not the destination node, it can further broadcast the RREQ packet to its neighbors.

3.1.2. Reverse Path Setup: As the RREQ is broadcasted to the various nodes across the network, it sets up a reverse path back from each of the nodes it is sent to, back to the source node by storing in each node, the address of the neighboring node it received the RREQ from.

3.1.3. Forward Path Setup: If the RREQ reaches a node with routing information about the destination node, it first verifies if the routing information is current or old by crosschecking the node sequence number of the destination node. If it is the same, then the information is current and it sends an RREP packet back to the source node. If the sequence numbers do not match, then the routing information is not current and the RREQ packet continues to be broadcasted to the neighbors of the present node.

3.1.4. Route Table Management: The purpose of this is that it acts as an RREQ expiration regulator. This helps to evade the reverse-path routing entries from those nodes that no longer exist on the path in between the source node and the destination node. The time for expiry depends completely upon the size of the ad-hoc network.

3.1.5. Path Maintenance: Every time the source node moves in the middle of an active period, it can re-start the path discovery process in order to establish a new path to the destination node. Also the mobility of the nodes that do not lie in the path between the source and destination, does not affect the routing at all.

\section{Motivation}

VANET is a highly researched and highly utilized technology that has numerous functions in multiple fields. As mentioned earlier, communication in VANETs takes place between different vehicles and roadside communication equipment. Hence, it is apparent that a lot of the information sent in VANETs may be tremendously important. However, when a lot of data has to be sent in a VANET between multiple source and destination nodes, a massive amount of data traffic may be generated in the network and eventually crash it. This loophole made it evident that there is a need for a well optimized routing protocol to be employed when implementing a VANET. This drove the research behind finding the appropriate routing protocol that could be applied and optimizing it in order to increase throughput and reduce the delay and the data traffic generated.

\section{Research Problem}

From above, we observe that AODV broadcasts its route request (RREQ) packets during the route discovery process which in turn, could lead to the accumulation of a large number of control packets in the network. As the control packets increase, there might be a lot of delay in the network due to internal collision of these control packets which in turn causes reduced throughput. In order to overcome these problems, our protocol focuses on controlling the control packets, which in turn can reduce the delay and lead to an increased throughput. 


\section{Methodology}

The scenario consists of nodes randomly moving in the network as defined by an Ad Hoc Network, hence modelling uncertainty in the network. Before the source can send a data packet to the destination, the route discovery process takes place. Although, this process is very similar to that of AODV, the ABAODV route discovery process makes use of an accumulator to accumulate the route request packets (RREQ) of a certain source node, hence reducing the number of control packets. During the route discovery process, each node sets up a temporary accumulator to accumulate the incoming RREQ packets that are broadcasted from every precedent node. This accumulator has a track of which node the RREQ packet is coming from as well as other routing information like the number of hops taken by the packet to reach the particular node, destination IP address, source IP address, broadcast IP address and many more. Once the accumulator accumulates these RREQ packets, the accumulator as a whole is broadcasted to its neighboring nodes. This in turn reduces the number of control packets being broadcasted across the network. In addition, if a node already has an accumulator, then a new accumulator is not created. The existing RREQ packets join the existing accumulator. This process continues till a path is found to the destination node. Once an accumulator reaches the destination node, the corresponding RREQ packet is dropped at this node while the rest of the accumulator continues its journey. The destination node unicasts a route reply (RREP) packet along the same path recorded by the RREQ packet back to the source. Once the RREP packet reaches the destination, based on the availability of the path (based on the timeout information) and the number of intermediate hops between the source and the destination (determined by the hop count), the data is sent along the determined route. The complete flow chart of the modified protocol is depicted in Diagram 1.

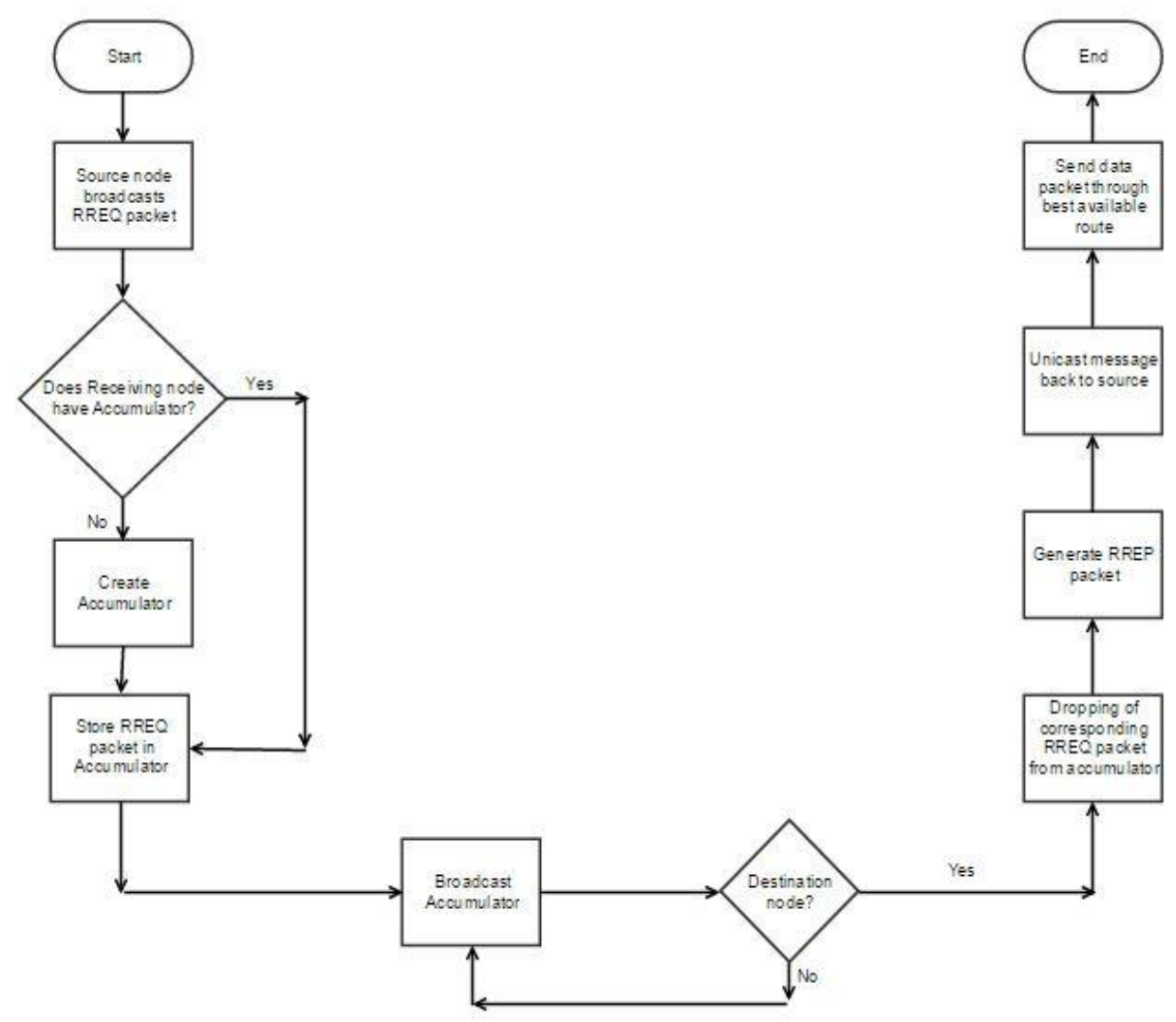

Diagram 1. Complete Flow of the ABAODV Routing Protocol 


\section{Simulation Model}

In this section, we will discuss the various tools that have been employed in simulating a real time vehicular network while evaluating the performance of the aforementioned tools. Also the methodology used to simulate the network has been clarified.

\subsection{Simulation Tools}

7.1.1. Simulation of urban mobility (SUMO): SUMO is a traffic simulator which is a microscopic and an open source simulation tool. This tool allows us to model each vehicle, its route and set various road parameters like the maximum speed and the number of lanes. Each vehicle is programmed to operate independent from each other. Apart from building a user defined road topology, SUMO also lets us import different real world maps processed using Open Street Maps as in this paper or even Google Earth.

7.1.2. Network Simulator 2 (NS2): NS2 is a network simulator that is an event driven and an object oriented tool which is widely used in the Networking community to study the dynamic nature of communication networks. The network simulator was first developed in 1989 by a group of researchers at the University of California, Berkeley; This tool has been further updated since then, with NS2 being the second version of the network simulator and the most widely used one. This tool can be used to simulate both wireless and wired protocols and functions. NS2 is programmed in OTcl at the front end and $\mathrm{C}++$ at the back end with both being linked by an OTcl interpreter. Furthermore, it generates an output file with a .nam extension which can be used to visualize the network on the Network Animator. Also, a trace file with a .tr extension is generated to evaluate various performance metrics and evaluate results.

\subsection{Simulation Scenario}

VANETs exhibit stronger challenges than other MANETs due to the highly dynamic movement of the vehicles. Also, in order to keep the simulation as realistic as possible, a realistic mobility model compatible with NS2 has to be developed to evaluate its performance. In addition to this, while typical MANET environment consists of generic open fields, VANET simulation environments would have to consider the obstacle that influence vehicular to vehicular communication like buildings, trees, roundabouts and many more.

This paper uses OpenStreetMap (OSM) to generate a realistic map scenario of a crowded area in the state of Chennai called Mambalam. This map is saved in OSM format which is then considered as a road map for the simulation in SUMO. The Figure 3 shows the extracted Open Street Map (OSM) of Mambalam, Chennai. 


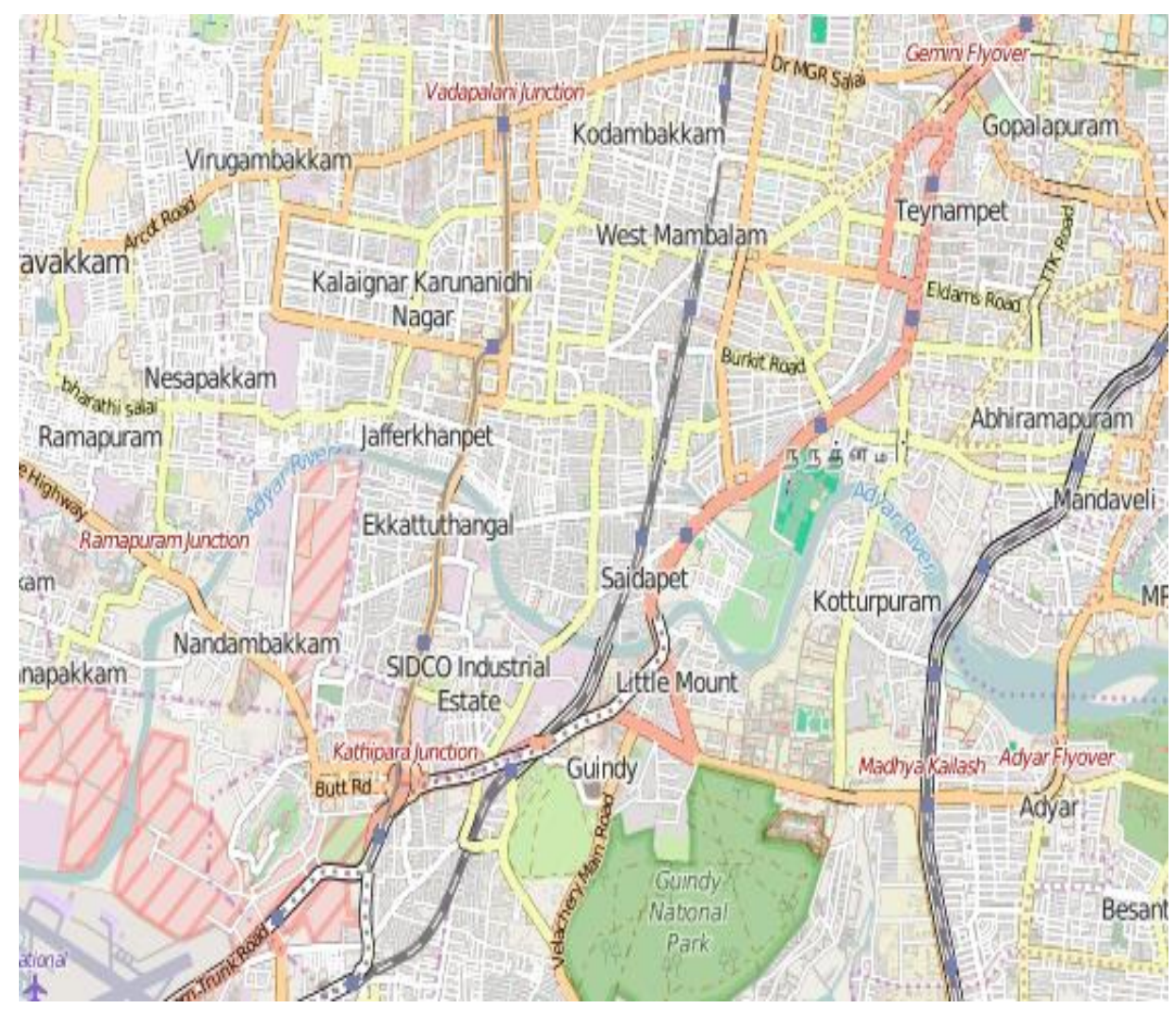

Figure 3. The Extracted OSM of Mambalam, Chennai

The road map is visualized using SUMO, setting the delay to $100 \mathrm{~ms}$ as shown in Figure 4 . We then obtain the $\mathrm{xml}$ files of the edges, nodes and network files from the road map, with the extension edg.xml, nod.xml and net.xml respectively. In addition, a file has to be created informing the simulator about the number of vehicles and the number of junctions, along with setting several other properties like road number, priority etc. This will in turn be used to generate a configuration file with an extension sumocfg. Before generating the trace file for the network simulation, we have to generate an fcd output, which is a trace file in the SUMO format. Finally, the trace file for the tr to be inputted to the network simulator is generated from the fcd output using the Trace Exporter. 

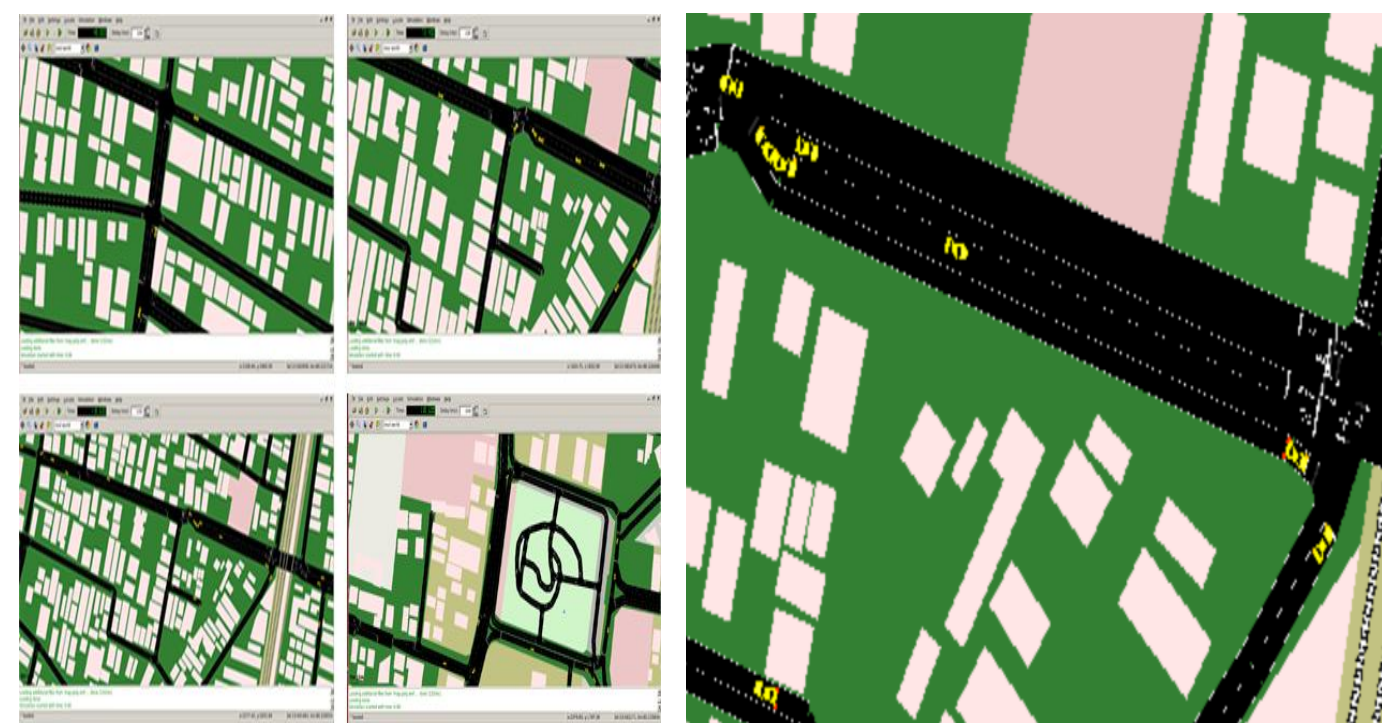

Figure 4. Visualization of the Road Map in SUMO

At this stage, the mobility and the traffic file is taken as input to the NS2 tcl file. The table 1 below, shows the configuration parameters assumed for the simulation:

Table 1. Configuration Parameters

\begin{tabular}{|c|c|}
\hline Parameter & Value \\
\hline Network Simulator & ns-2.35 \\
\hline Channel Type & Wireless \\
\hline Network Interface type & Physical Wireless \\
\hline Routing Protocol & ABAODV, AODV \\
\hline Interface Queue type & Priority Queue \\
\hline Queue Length & 50 packets \\
\hline Number of nodes in the topography & $5,20,40,60,80,100,120$ \\
\hline Tx Range & $400 \mathrm{~m}$ \\
\hline Simulation Time (s) & 30 \\
\hline MAC Protocol & IEEE 802.11 \\
\hline Computer & i7 processor, 6GB RAM \\
\hline Operating System & Ubuntu \\
\hline
\end{tabular}

In the experiments, the simulation model has been created using ns-2.35. Performance metrics have been measured with respect to both the ABAODV and the AODV protocol. For both the protocols, 30 seconds has been set as the simulation period. The queue length has been set to 50 packets while the size of each packet has been set to 512 bytes. The entire experiment has been done in the Ubuntu - 12.04 operating system.

The complete simulation scenario is depicted in the architecture for VANET simulation as shown in Figure 5. 


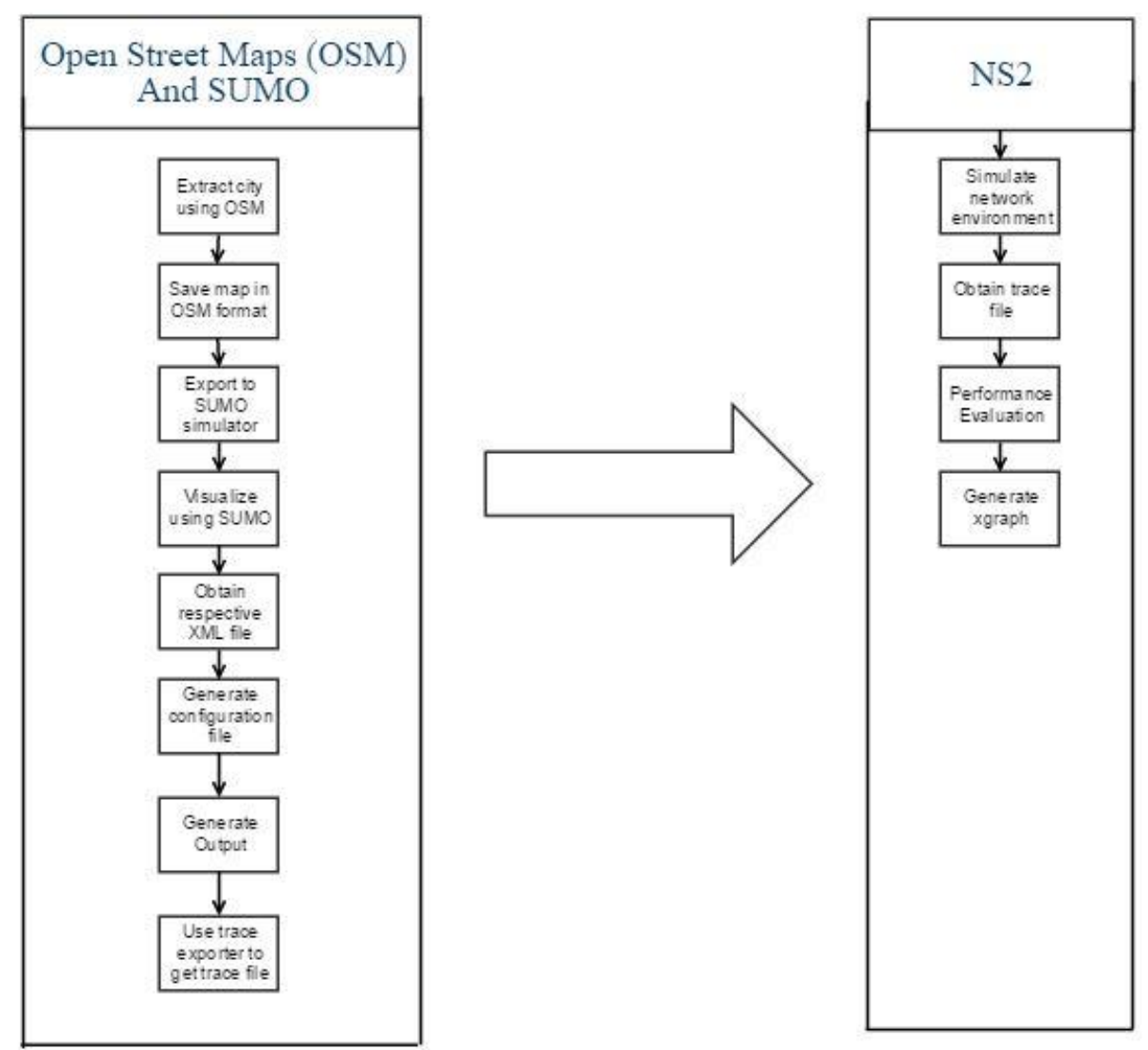

Figure 5. Simulation Architecture for VANETs

\section{Performance Evaluation}

This section presents the comparison of ABAODV, AODV and DSR, simulated using NS2, with respect to the performance metrics given in the first section. The second section presents the evaluation and elucidation of the results obtained from the simulation, in the form of graphs; generated using MATLAB.

\subsection{Performance Metrics}

The below 2 are the metrics used for performance evaluation of the two protocols:

- Delay: The delay of a network specifies the amount of time in seconds, taken by a correctly received packet to travel across the network from the source to destination.

- $\quad$ Average Throughput: It is the average of the total number of packets received at the destination over simulation time multiplied by the number of nodes in the network.

\subsection{Performance Results}

Figure 6.1 shows the comparison of the delay of the ABAODV, AODV and DSR protocols with respect to time. The $\mathrm{X}$ axis denotes the time in seconds while the $\mathrm{Y}$ axis represents the delay of the network. The number of nodes taken in the simulation scenario was 5 nodes. 


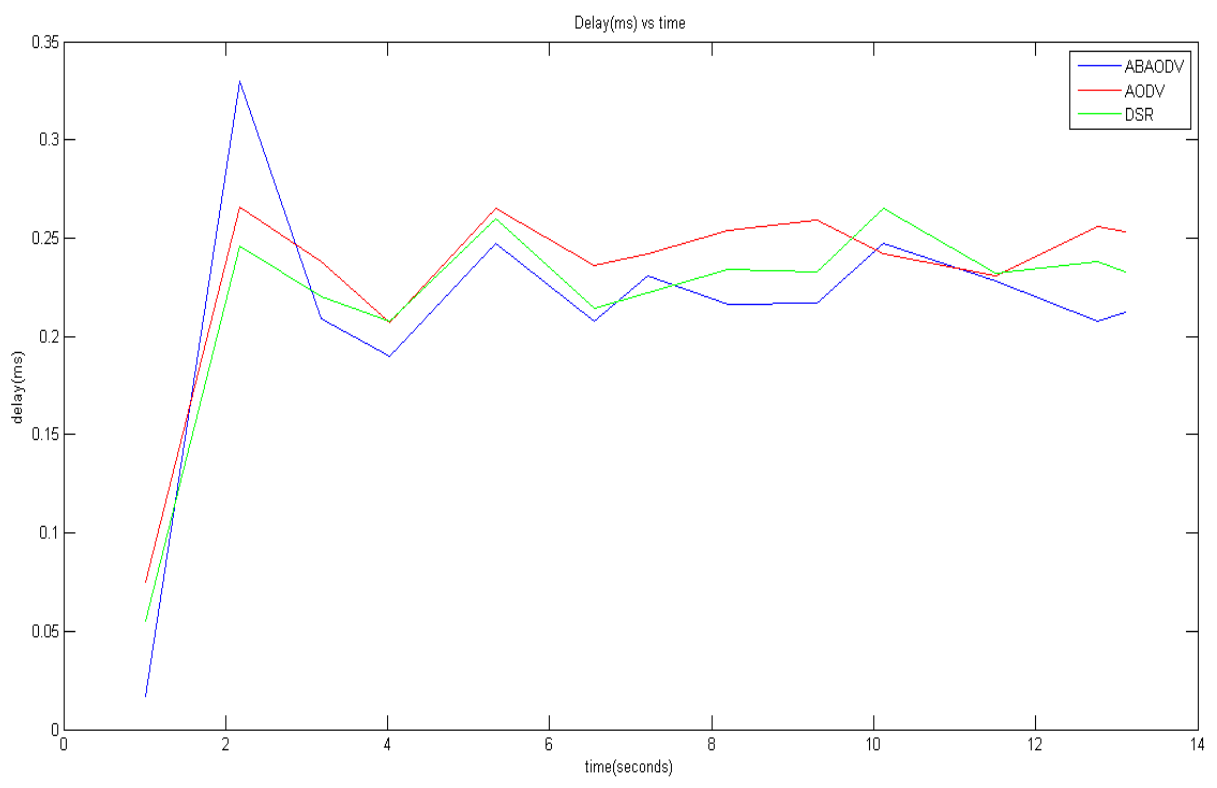

Figure 6.1. Delay of Packets with respect to Time

From Figure 6.1 and Table 2.1, we can infer that none of the protocols clearly outperforms the others. There is a time where ABAODV has lesser delay and vice versa (At $\mathrm{t}=2.17 \mathrm{~s}, \mathrm{t}=10.122 \mathrm{~s}, \mathrm{t}=7.217 \mathrm{~s}$ ). This can be explained by the fact that, at the start, the ABAODV with an empty accumulator behaves almost in the same way as AODV and DSR which broadcasts its RREQ packets. As time progresses, we notice that the delay of ABAODV reduces further below that of AODV and DSR, due to the clear reduction in the number of control packets in the network. This in turn reduces the number of collisions within the network, hence reducing delay. At times where AODV and DSR have less delay than that of ABAODV, we can say that this is a point where there are less number of packets in the accumulator, hence the overhead in the network is much smaller than that of AODV. Overall, we can say that at most of the times, packets using the ABAODV protocol takes less time to reach its destination than the case when it uses the AODV or DSR protocol.

From Figure 6.1 and Table 2.1, we can infer that none of the protocols clearly outperforms the others. There is a time where ABAODV has lesser delay and vice versa (At $\mathrm{t}=2.17 \mathrm{~s}, \mathrm{t}=10.122 \mathrm{~s}, \mathrm{t}=7.217 \mathrm{~s}$ ). This can be explained by the fact that, at the start, the ABAODV with an empty accumulator behaves almost in the same way as AODV and DSR which broadcasts its RREQ packets. As time progresses, we notice that the delay of ABAODV reduces further below that of AODV and DSR, due to the clear reduction in the number of control packets in the network. This in turn reduces the number of collisions within the network, hence reducing delay. At times where AODV and DSR have less delay than that of ABAODV, we can say that this is a point where there are less number of packets in the accumulator, hence the overhead in the network is much smaller than that of AODV. Overall, we can say that at most of the times, packets using the ABAODV protocol takes less time to reach its destination than the case when it uses the AODV or DSR protocol. 
Table 2.1. Delay of Packets with respect to Time

\begin{tabular}{|l|l|l|l|}
\hline Time (Seconds) & $\begin{array}{l}\text { ABAODV - Delay } \\
\text { (ms) }\end{array}$ & $\begin{array}{l}\text { AODV - Delay } \\
(\mathbf{m s})\end{array}$ & $\begin{array}{l}\text { DSR - Delay } \\
(\mathbf{m s})\end{array}$ \\
\hline 1.017 & 0.017 & 0.075 & 0.055 \\
\hline 2.17 & 0.33 & 0.266 & 0.246 \\
\hline 3.185 & 0.209 & 0.238 & 0.220 \\
\hline 4.03 & 0.19 & 0.201 & 0.208 \\
\hline 5.336 & 0.247 & 0.265 & 0.260 \\
\hline 6.551 & 0.208 & 0.236 & 0.214 \\
\hline 7.217 & 0.231 & 0.242 & 0.222 \\
\hline 8.199 & 0.216 & 0.254 & 0.234 \\
\hline 9.304 & 0.217 & 0.259 & 0.233 \\
\hline 10.122 & 0.247 & 0.242 & 0.265 \\
\hline 11.508 & 0.228 & 0.231 & 0.232 \\
\hline 12.766 & 0.208 & 0.256 & 0.238 \\
\hline
\end{tabular}

Figure 6.2. Shows the total throughput of the ABAODV, AODV and DSR protocol with respect to the number of nodes. While the $X$ axis consists of the number of nodes in the network, the $\mathrm{Y}$ axis denotes the total throughput in each of these scenarios. The network simulation scenario considers $20,40,60,80,100$ and 120 nodes for performance evaluation.

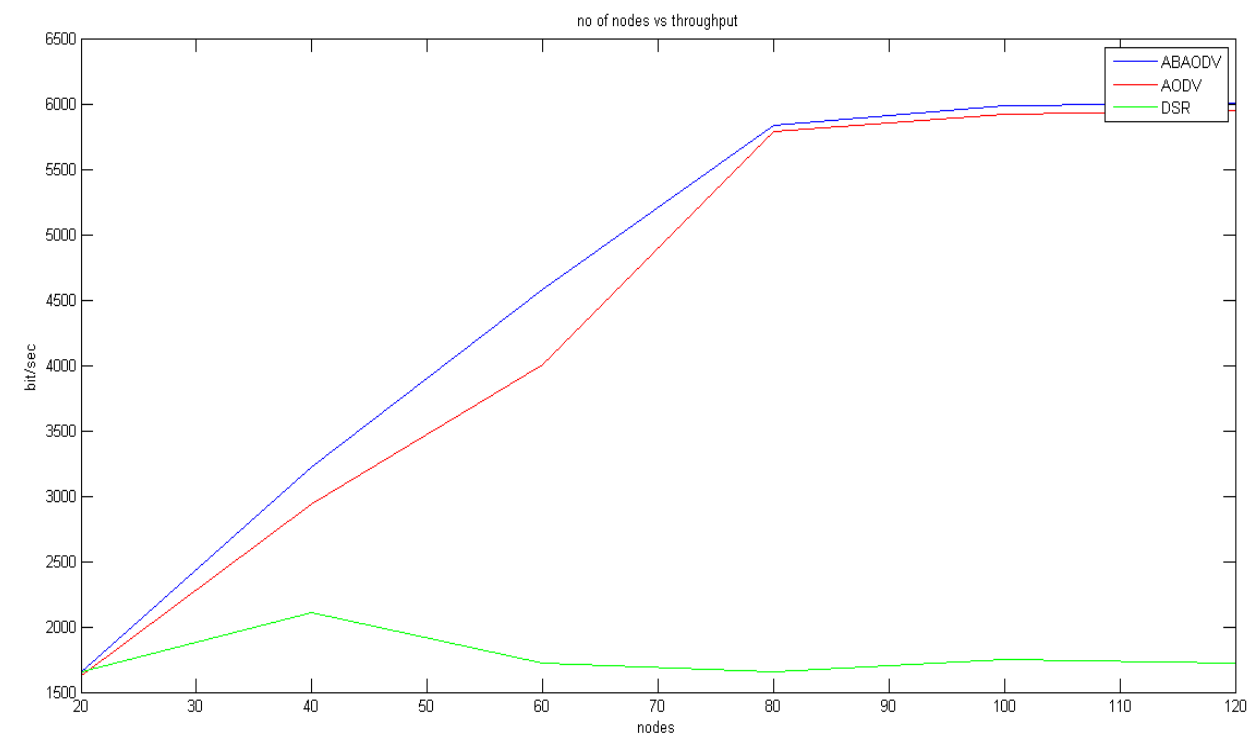

\section{Figure 6.2. Total Throughput of the Network with Respect to the number of Nodes}

From Figure 6.2 and Table 2.2, it can clearly be inferred that ABAODV outperforms the AODV and DSR protocol with respect to the throughput in the network. We realize that as the number of nodes in the network keep increasing, AODV and DSR broadcasts more number of RREQ packets in the network, the number of control packets is humongous, the larger the possibility of collision within the network, hence larger the possibility of packet loss as compared to the accumulator style broadcast of ABAODV which controls the number of control packets in the network, hence resulting in a greater throughput. 


\section{Table 2.2. Total Throughput of the Network with respect to the Number of Nodes}

\begin{tabular}{|c|c|c|c|}
\hline $\begin{array}{c}\text { Number } \\
\text { of } \\
\text { nodes }\end{array}$ & $\begin{array}{c}\text { ABAODV - Average } \\
\text { Throughput } \\
\text { (bits/sec) }\end{array}$ & $\begin{array}{c}\text { AODV-Average } \\
\text { Throughput } \\
\text { (bits/sec) }\end{array}$ & $\begin{array}{c}\text { DSR-Average } \\
\text { Throughput } \\
\text { (bits/sec) }\end{array}$ \\
\hline 20 & 1651 & 1621 & 1653 \\
\hline 40 & 3220 & 2934 & 2109 \\
\hline 60 & 4578 & 4009 & 1721 \\
\hline 80 & 5834 & 5791 & 1653 \\
\hline 100 & 5983 & 5920 & 1752 \\
\hline 120 & 6007 & 5951 & 1719 \\
\hline
\end{tabular}

In addition to this, we realize that the DSR protocol's total throughput degrades as the number of nodes in the network increase. This is possibly due to the usage of stale routes. Hence, the number of packets received at the destination node with respect to the number of nodes, is more using the ABAODV protocol compared to the AODV protocol.

\section{Conclusion \& Future Scope}

In this paper, a new routing protocol for Vehicular Ad Hoc networks has been developed, which is a modified AODV protocol, by making an extension to the existing AODV protocol. Simulation is carried out in a realistic simulation environment using OpenStreetMap and SUMO. Results show that ABAODV has lesser delay compared to both the AODV and DSR protocols at most of the times during the middle of the simulation i.e. when the accumulator has a sufficient number of RREQ packets. Moreover, ABAODV clearly outperforms AODV and DSR with respect to the total throughput. As the number of nodes increase, ABAODV significantly reduces the number of control packets in the network with the help of the accumulator, as compared to the large number of control packets in the network in terms of AODV and DSR.

In the future, it would be interesting to see how ABAODV performs when implemented in the single mobility model and manually generated maps. Also its performance can be analyzed with respect to a lot of other performance metrics like packet delivery ratio, routing overhead and average network load. These metrics can be measured for different flow of traffic.

\section{References}

[1] A. Abolfazl, M. Soruri and Khosrozadeh, "A New AODV routing protocol in Mobile Adhoc Networks", World Applied Sciences Journal, vol. 19, no. 4, (2012).

[2] A. Jardosh and E. B. Royer, "Toward realistic mobility models for mobile ad hoc networks", Proceedings of the $9^{\text {th }}$ Annual International Conference on Mobile Computing and Networking (MobiCom 2003), (2003).

[3] C. Perkins and E. Royer, "Ad Hoc on demand Distance vector routing", IETF, RFC 3561, (2003).

[4] D. Helbing, "Traffic and Related Self-driven Many-particles Systems", Rev. Modern Physics, vol. 73, (2001), pp. 1067-1141.

[5] H. Saini and R. M. Patna, "Implementation and Performance Analysis of AODV Routing Protocol in VANETs", vol. 2, iss. 3, (2014).

[6] J. Broch, "A Performance Comparison of Multi-Hop Wireless Ad Hoc Network Routing Protocols", Proceedings ACM MOBICOMM 98, Dallas, TX, (1998).

[7] J. Haerri, M. Fiore, F. Filali, C. Bonnet, C. F. Chiasserini and C. Casetti, "A Realistic Mobility Simulator for Vehicular Ad Hoc Networks", Eurecom Technical Report, Institut Eurecom, France, (2005).

[8] J. Haerri, F. Filali and C. Bonnet, "A Framework for Mobility Models Generation and its Application to Inter-Vehicular Networks", $3^{\text {rd }}$ IEEE International Workshop on Mobility Management and Wireless Access (MobiWac'05), Maui, Hawaii, U.S.A., (2005). 
[9] K. Bhaghchandani and Y. M. Sharma, "Exploration of VANET Mobility Models with New Cluster Based Routing Protocol", International Journal of Soft Computing and Engineering (IJSCE), vol. 2, iss. 6, (2013), pp. 2231-2307.

[10] K. C. Lee, U. Lee and M. Gerla, "Survey of Routing Protocols in Vehicular Ad Hoc Networks", Advances in Vehicular Ad-Hoc Networks: Developments and challenges references, IGI Global, 2010, (2013), pp. 149-170.

[11] M. Abolhasan, T. Wysocki and E. Dutkiwicz, "A review of routing protocols for mobile ad hoc networks", Ad Hoc Networks, vol. 2, no. 1, (2004), pp. 1-22.

[12] M. Altayeb and I. Mahgoub, "A survey of Vehicular Ad hoc Networks Routing Protocols", International Journal of Innovation and Applied Studies, ISSN 2028-9234, vol. 3, no. 3, (2013), pp. 829-846.

[13] NS2. Network Simulator - ns-2. Available at \{http://nsnam.isi.edu/nsnam/index.php/Main_Page , (2013).

[14] P. Johansson, "Scenario-based Performance Analysis of Routing Protocols for Mobile Ad-Hoc Networks”, Proceedinga IEEE/ACM Mobicom'99, Seattle, WA, (1999), pp. 195-206.

[15] R. A. Santos, "Performance Evaluation of Routing Protocols in Vehicular Ad Hoc Networks", International Journal of Ad Hoc and Ubiquitous Computing 2005, vol. 1, no.1/2, pp. 80-91.

[16] R. K. Aswed and M. A. Abdala, "Analyzing Routing Protocols Performance in VANET Using 802.11p and 802.11g", vol. 5, iss. 1, (2015), pp. 6-12.

[17] S. R. Das, "Comparative Performance Evaluation of Routing Protocols for Mobile Ad-Hoc Networks", In $7^{\text {th }}$ Int. Conf. on Comp. Communication and Networks, Lafayette, LA, (1998), pp. 153-161.

[18] SUMO - Simulation of urban mobility. Available at http://sumo.sourceforge.net/, (2013).

[19] S. Jaap, M. Bechler and L. Wolf, "Evaluation of Routing Protocols for Vehicular Ad Hoc Networks in City Traffic Scenarios", Proceedings of the $5^{\text {th }}$ International Conference on Intelligent Transportation Systems Telecommunications (ITST), Brest, France, (2005).

[20] T. Camp, J. Boleng and V. Davies, "A Survey of Mobility Models for Ad Hoc Network Research", Wireless Communications and Mobile Computing, vol. 2, iss. 5, (2002), pp. 483-502.

[21] T. H. Clausen and P. Jacquet, "Optimized Link State Routing (OLSR)”, RFC 3626, (2003).

[22] T. H. Clausen, P. Jacquet and L. Viennot, "Comparative Study of Routing Protocols for Mobile Ad-Hoc Networks", $1^{\text {st }}$ IFTP MedHocNet Conference, (2002).

[23] The FleetNet Project, http://www.et2.tu-harburg.de/, fleetnet/English/vision.html.

\section{Authors}

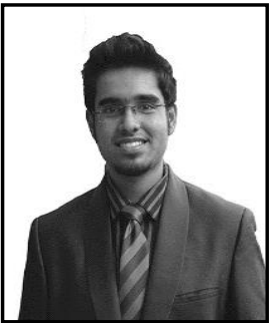

Anush Baskaran, he is with the School of Computer Science and Engineering at VIT University, Vellore, India. His research interests lie in Wireless Ad Hoc Networks, Information Security, Network Security and Data Centric Networks. He is currently executing many projects in the same.

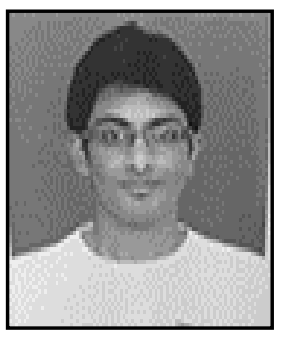

Sushant Ramesh, he is a student, pursuing a Bachelors of Technology in Computer Science and Engineering at VIT University, Vellore, Tamil Nadu, India. He has high enthusiasm and ambition towards the projects he works on. His academic interests include Artificial Intelligence (primarily Fuzzy Logic and Artificial Neural Networks) and Embedded Systems.

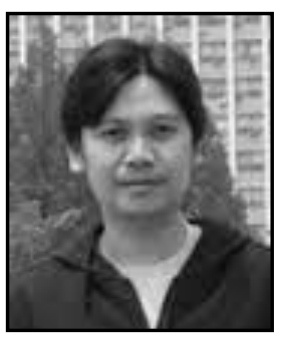

Ronnie D. Caytiles, he had his Bachelor of Science in Computer Engineering- Western Institute of Technology, Iloilo City, Philippines, and Master of Science in Computer Science- Central Philippine University, Iloilo City, Philippines. He finished his Ph.D. in Multimedia Engineering, Hannam University, Daejeon, Korea. Currently, he serves as an Assistant Professor at Multimedia Engineering department, Hannam University, Daejeon, Korea. His research interests include Mobile Computing, Multimedia 
Communication, Information Technology Security, Ubiquitous Computing, Control and Automation

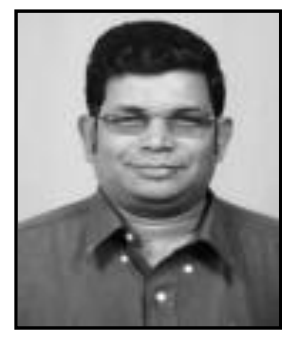

N. Ch. S. N. Iyengar, he is a Professor, SCS Engineering at VIT University, Vellore, TN, India. His research interests include Distributed Computing, Information Security, Intelligent Computing, and Fluid Dynamics (Porous Media). He had much teaching and research experience with a good number of publications in reputed International Journals \& Conferences. He chaired many Intl. Conf. \& delivered Key note and Invited lectures,. He is Editorial Board member for many Int'l Journals like Cybernetics and Information Technologies (CIT)- Bulgaria, Egyptian Computer Science Inderscience etc., Also Editor in Chief for International Journal of Software Engineering and Applications (IJSEA) of AIRCC, Advances in Computer Science (ASC) of PPH. 
International Journal of Future Generation Communication and Networking Vol. 9, No.7, (2016) 